\title{
Tangible Lighting Proxies: Brokering the Transition from Classroom to Stage
}

\author{
Rebecca Nicholson \\ Open Lab \\ Newcastle University \\ Newcastle upon Tyne, UK \\ r.nicholson5@newcastle.ac.uk
}

\author{
David Kirk \\ Open Lab
}

Newcastle University

Newcastle upon Tyne, UK

david.kirk@newcastle.ac.uk
Tom Bartindale
Action Lab

Monash University

Melbourne, Australia

tom.bartindale@monash.edu

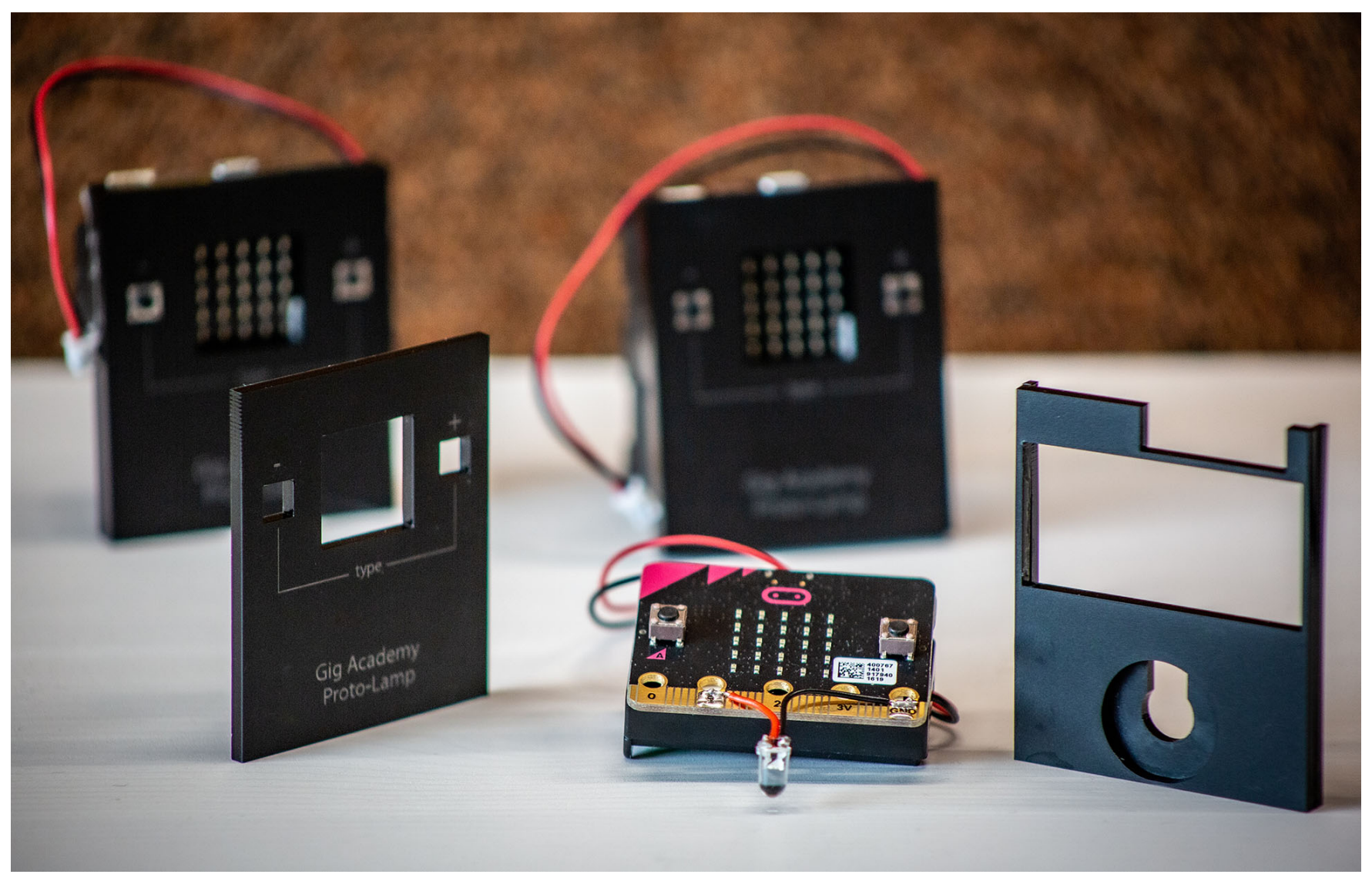

Figure 1: Tangible Lighting Proxies

\section{ABSTRACT}

Supporting Practice-based vocational skills development in the classroom can be logistically challenging, however tangible interfaces present natural affordances for supporting such skill development. In the context of teaching stage lighting specifically, we present an open source toolkit based around tangible hardware

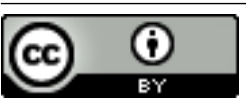

This work is licensed under a Creative Commons Attribution International 4.0 License. TEI '21, February 14-17, 2021, Salzburg, Austria ACM ISBN 978-1-4503-8213-7/21/02.

https://doi.org/10.1145/3430524.3440659 proxies to support the teaching of practice-based skills. Our tangible proxies embody key configuration, interaction and optical properties of real stage equipment. Drawing on notions of representation and bi-directional digital-to-physical transformation, we design a toolkit that specifically supports a gradual transition between virtual, simulated and real equipment during the learning journey, and open the door to embedding stage craft into schools. Through reporting on deployments in two schools, we discuss the affordances of such proxies and their potential for supporting the teaching and learning of practice-based skills in the classroom. 


\section{CCS CONCEPTS}

- Human-centered computing $\rightarrow$ Human computer interaction (HCI); Interaction devices; • Applied computing $\rightarrow$ Performing arts; Interactive learning environments.

\section{KEYWORDS}

Tangible; TUI; Hardware Proxies; Stage lighting; Practice-based skills

\section{ACM Reference Format:}

Rebecca Nicholson, David Kirk, and Tom Bartindale. 2021. Tangible Lighting Proxies: Brokering the Transition from Classroom to Stage. In Fifteenth International Conference on Tangible, Embedded, and Embodied Interaction (TEI '21), February 14-17, 2021, Salzburg, Austria. ACM, New York, NY, USA, 13 pages. https://doi.org/10.1145/3430524.3440659

\section{INTRODUCTION}

The teaching of stage lighting in the K-12 Creative Arts classroom can provide learners with the opportunity to engage in STEAM (a movement to embed Science, Technology, Engineering, and Mathematics teaching within the Arts) [25] something which is currently a topic of increasing interest to the HCI community [23]. While the Creative Arts are widely taught within schools, the teaching of technical roles is not commonly included [34] because of the constraints of the K-12 classroom. Clear barriers exist to supporting students interacting with heavy, bulky, potentially dangerous, and expensive equipment that is hard to setup in a classroom. Tangible interfaces, however, provide opportunities to engage students with learning about technically-complex contexts in the classroom, the potential of which is well documented within the TEI community e.g. $[1,3,26,27]$. Notably, tangibles provide opportunities to teach practice-based vocational skills in a manageable setting [36].

The notion of representation is key to the design of such tangible interfaces to effectively mediate interaction and cognition within the learning environment [31]. Price's existing conceptual framework for representation in tangible learning environments outlines several features of digital manipulatives (location, dynamics, correspondence, and modality) with which to consider different representation-artifact relationships and how they impact learning. These have been applied in several contexts to understand the implications of design decisions on the potential for tangibles to be used in the classroom and other learning scenarios [24, 32]. As yet, this framework has been applied primarily in contexts where representations of abstract concepts are being designed and evaluated. While we are beginning to understand the role of representations in such contexts, their role in learning scenarios where tangibles simulate existing real-world physical artifacts is unclear. For example, stage lighting poses a challenge as students require opportunities for authentic learning in settings where there are clear logistical and safety concerns.

Drawing on the affordances of tangible interfaces for classroom learning, knowledge that representation is key, and in response to the practical challenges of teaching in a classroom, we developed a toolkit to support students' practical experience with stage lighting equipment through the use of equipment 'proxies'. These tangible proxies embody key configuration, interaction and optical properties of real lighting equipment and venue space that would otherwise be inaccessible to students, but that can be practically used in a classroom setting. Rather than virtually simulate an environment these proxies support incremental experimentation and practice of skills by supporting learners' transition between simulations and real equipment through the use of a 'broker'. The toolkit can therefore be configured to focus on specific elements of stage lighting without students needing consistent access to a complete (and real) lighting setup.

In this paper we contribute the concept of tangible proxies as a viable way to support the teaching of practice-based vocational skills within a K-12 classroom. We present our open source implementation of a tangible toolkit to teach stage lighting, including these proxies and report on deployments of our prototype in two UK schools. Specifically we draw out considerations for designing tangible interfaces that allow transition from 'real' but impractical teaching scenarios to classroom settings by supporting bi-directional digital to physical transforms, something which is as yet under researched [29]. Drawing on these experiences, we discuss how tangible proxies can be designed to engage students with practice-based learning and contribute considerations for the design of such proxies to support learning skills that that are otherwise impractical or inaccessible in schools.

\section{BACKGROUND AND RELATED WORK}

\subsection{Teaching Stage Lighting}

As with many mixed-discipline roles within the Performing Arts, stage lighting is a practice-based skill [45] typically learned through repetitive hands-on experience with physical artifacts and specifically configured tools [42]. Supporting trial-and-error and learning through experimentation are key to enabling both conceptual and practical learning. Typically this experience is gained through vocational training programs, most commonly through a masterapprentice relationship within a community of practice [47] where requisite knowledge is built through hands-on repetitive experience with the equipment and processes utilized within the industry. Within this community of practice, students typically engage in "Deliberate Practice" [9], a cycle of highly structured activities and immediate informative feedback, the explicit goal of which is to improve performance. This is key in the acquisition of performative expertise more than simply gaining hours of experience. However, supporting such practice is resource intensive in terms of both educators and equipment [10], limiting the number of students who have access to these opportunities. Although specialist stage lighting curricula are taught worldwide post-16, these courses are almost exclusively offered by creative arts or performance focused institutions [34]. Such specialist institutions are naturally configured for hands-on interaction with industry standard equipment and venues [39], specifically because it allows for experiential [22] and embodied [38] models of learning, as is typical within the industry. This model of teaching is clearly not suitable for a resource constrained setting such as a K-12 classroom, where factors such as the length of classes (equipment setup is time consuming), access to resources, health and safety concerns and staff expertise often preclude the teaching of stage lighting. Teachers are reticent to (and often legally precluded from) letting students interact with 
expensive and potentially dangerous equipment, even with supervision. This is compounded by the lack of basic knowledge that teachers often have in this area, the time required to configure the related hardware as well as their physically large size and weight.

\subsection{Using Simulations}

In many cases, merely providing a physical representation of a scenario in which skills are acquired is valuable to learning [17] This has been documented particularly in the context of distributing cognition within group learning settings [46]. Within stage craft, this technique is often deployed for set-design [20], and stage layout. When working with the lighting designer however, these models are only used as a shared representation of the space and its constraints. As such, they afford high-level shared cognition of the problem space, but lack the opportunity for specific skills learning. Within the stage lighting domain virtual simulations have been more regularly used due to the complex nature of representing stage-lighting in physical models or simulations.

While tangible interfaces offer clear advantages for students in the learning environment, it is worth noting (and learning from) how the industry has responded to comparable challenges, particularly in access to equipment and training. Their response to this complexity has been to develop software-based simulators which allow for pre-configuration of settings, allowing lighting designers to experiment with different configurations without using real equipment. These simulators are roughly split into two types: Visualizers which make use of recent advances in computational rendering of lighting effects to create editable and navigable 3D visual representations of the venue such as Capture ${ }^{1}$ or ShowXpress ${ }^{2}$; Planning tools which offer 2D or abstract representations of the setup for planning and manipulation such as ENTTEC D-Pro ${ }^{3}$ or Lightkey ${ }^{4}$ Since much of this equipment is situated in venues with limited access, some vendors also offer simulators of their specific control equipment $t^{5}$ to aid in engineer training.

The majority of these tools are however designed with the professional lighting designer in mind. They simulate and extend the functionality of complex control equipment, and although designed to aid in experimentation, they assume pre-existing knowledge of basic lighting fundamentals and equipment, and do not easily support a novice gaining experience or the teaching of underlying concepts. Although projects such as ThorDMX [2] have sought to lower the complexity barrier to novice creation of custom lighting controllers, such interfaces do not translate well into the classroom since they are designed specifically for an individual interaction paradigm - as seen with the master-apprentice model.

\subsection{Tangibles for Learning}

The process of learning stage lighting involves both an abstract cognitive understanding of the underlying concepts, as well as deliberate practice with specific pieces of industry standard equipment.

\footnotetext{
${ }^{1} \mathrm{http}: / /$ www.capture.se

${ }^{2}$ https://www.chauvetdj.com/showxpress/

${ }^{3}$ https://www.enttec.com.au/product/software-products/lighting-control-

software/d-pro/

${ }^{4}$ https://lightkeyapp.com/en/

${ }^{5}$ https://zero88.com/zeros
}

2.3.1 Abstract Representation. The potential for tangibles to support the learning of abstract or conceptual topics through simulation is well documented [43]. They have been used in a variety of contexts such as language learning [16], computing [49] as well as computational thinking [11]. One existing framework for tangibles in learning contexts [31] places a focus on artifact-representation, presenting four features for consideration: location - describing the coupling between physical and digital artifacts, dynamics - describing the information associations between artifact and representation, correspondence - describing the degree to which the physical properties of the objects are mapped to the learning concepts, and modality - describing the different representation modalities.

It is therefore clear how we might use tangibles to represent specific abstract concepts - such as how lighting control protocols operate [8], or the behavior of light [44]. In these contexts it is clear that to support the learning of underlying concepts, tangible systems need to allow for, or prompt, reflection on these concepts [6]. One way to do this is to allow for a model of shared cognition which offers clear opportunities for cycles of action and reflection as students alter the state of the model and then reflect on the outcome of that change [33] referred to by Schön as 'reflection in action' and something aspired to in many learning contexts [37].

2.3.2 Supporting Deliberate Practice. Although the above framework is helpful to design new tangibles that map learning outcomes to abstract concepts, the literature is less clear how we should map tangible representations to concrete, real world interactive objects such as stage equipment. In our case, alongside conceptual learning we need to encourage deliberate practice of specific practice-based skills usually conducted with real equipment (rather than simulations). Examples of tangibles for the practice of such skills are less common though there are examples such as Tinkertable [36] for learning about warehouse logistics as well as for learning about industrial refrigeration maintenance [41]. Consideration of artifactrepresentation relationships is still fundamental to the design of such a system as we need to understand the potential relationship between simulations and the real artifacts they are representing. We do know that when designing physical representations, learning outcomes can be greatly influenced by the design of the relationship between the tangible artifact and the specificities of the learning domain [7]. This could translate to us mapping the key optical and configuration properties of lamps which distinguish them from each other, while placing less focus on the physical appearance or size of the model [40], thus defining the physical and representational correspondence required to support learning.

Traditionally, tangible interfaces for simulated learning are designed with the tangible being the primary input modality, taking advantage of the ability for the physical to transform the digital [35] (although there are some notable exceptions outside of learning [30]). However, is has been noted that the transform from digital to physical is less understood [29] and infrequently applied due to the complexity of designing specific (plausible) tangibles that can be manipulated. In the case of stage equipment however, physical artifacts already exist that can be manipulated digitally (i.e. real stage lights), and so our challenge is to integrate these representations into a tangible system that supports transforms between abstract tangible artifacts and the real thing. Once these transforms exist to 
support practice-based learning, students also require a mechanism to translate abstract concepts to the real stage environment.

2.3.3 The Challenge of Transference. Existing research has highlighted that supporting students moving easily from learning with a tangible system to a 'real-world' scenario is difficult $[6,18]$. This transition is known as 'transference' [13], where it is suggested that learners acquire an 'action schema responsive to the affordances' of the learning situation. Transferring knowledge would then be possible if the target situation had the same affordances as the real-world scenario. We can draw lessons from from similar school settings that leverage abstract representations (albeit not tangible) to teach real-world concepts such as in learning to program. In work with block-based code environments, it has been demonstrated that students' ability to manipulate an interface does not necessarily correlate to their understanding of the underlying concepts [14] Supporting a bi-directional transition, however, between blocks and code [28] has been successful in supporting student's cognitive transference of underlying concepts to authentic programming environments.

\section{DESIGN CHALLENGES}

Based on current pedagogy in institutions where stage lighting is taught and industry sector practice, we aimed to design a toolkit that supported the teaching of practice-based stage lighting skills in the classroom. Drawing on existing literature we knew that any resource we designed needed to support: i) key elements of traditional vocational learning, ii) skills development outside of the primary context, iii) the use of the 'simulation' within the K-12 specific context, and iv) the transition from 'simulation' to 'real' equipment.

\subsection{Supporting Vocational Learning}

We knew that we needed to support the key elements of traditional vocational learning typically provided through a master and apprentice model. Research in similar practice-based domains such as medicine [21] make it clear that whatever we created needed to: i) support sustained, deliberate practice within a safe environment, ii) map onto real-life experience, and iii) provide a supportive, learner-centered way of learning.

\subsection{Skills Development Outside the Primary Context}

As the toolkit is for use in the classroom, we also needed to support the three major components identified by Grantcharov et al. [12] for supporting skills development outside of the primary context: i) build cognitive knowledge of the steps required and the function and operation of the equipment; ii) Offer instruction in basic generic and fundamental skills that will enable students to actually perform the required tasks; iii) Provide opportunities to perform these skills in a variety of platforms.

To teach stage lighting this meant we needed to support students in learning a set of core procedural steps and facilitate them practicing these steps on a variety of lighting equipment and software. Core procedural steps for lighting are [34]:
(1) Plan: Plan the intended lighting states (how the stage should look) required for the event, making as much reuse of lamps (lights) as possible. In this step, building a mental model of the venue, optical properties of particular lights and the physical space is key.

(2) Rig: Select and mount lamps to the venue and route power and control cabling. This step involves understanding the specific physical properties of each piece of equipment and how they can be utilized to produce the plan according to the mental model.

(3) Configure: Set addresses to lamps so that they can be controlled. This involves coordinating with other members of the production team, and a conceptual understanding of control procedures.

(4) Focus: Adjust lamps (position, focus, color, shutters, etc.) to the correct attitude in the venue. A physical process of operating controls on lights to produce the desired optical effects.

(5) Program: Program repeated or complex states into the control desk and/or rehearse timings. A process of building practical skill with specific equipment.

Each of these steps requires the understanding and application of both knowledge (i.e. of the properties of each type of lamp) and skills (i.e. best practice for addressing lamps, or performing focusing) [5]. For students to practice these skills we needed to ensure that certain fundamentals of stage lighting equipment were modeled. Stage lights are controlled via dimmer switches in a similar way to domestic homes. These are usually remote-controlled using DMX512 (DMX), an industry specific one-directional control protocol. Thus we needed to ensure that (as a minimum) certain features of this protocol were modeled including: Channels - DMX protocols work in groups of 512 channels, referred to as a universe. Each channel is capable of being set to any 8-bit value (0-255). Lights are controlled by transmitting individual values between the controller (lighting desk) and lights (lamps) to control individual properties. Some lamps take up one channel (those that have a single dimmable bulb), while others will have other properties such as color or other effects that require more channels. A particular lamps mapping from channels to physical control properties is referred to as its personality. RGB color mixing - lamps which are capable of changing between red, green and blue color (RGB) usually require at least three channels - one to control red, one for green and one for blue. To achieve other colors requires knowledge of how to 'mix' light to achieve these colors. Addressing - To 'fit' all lamps into the 512 channels in a universe, each is given a starting address at which to start listening to channel data. Lamps with identical personalities can be configured to listen to the same address.

\subsection{Support Learning Specifically Within the K-12 Classroom}

There are notable temporal and situational constraints of typical school environments that we needed to account for in our design. In most classrooms, lessons must be conducted within a short time window (typically between 50-70 mins), in a shared (and physically small) classroom space. While the space can be configured according to the class requirements, it needs to be reconfigured back to a 
traditional teaching environment when a lesson finishes. While using school event spaces are one solution to this, it may still not be feasible due to questions about practicality: for example, equipment may not be permanently setup or teacher supervision time may be limited. Finally, there are significant health and safety concerns in students using pieces of large and heavy stage equipment, many of which are connected to high-power transformers as well as legal constraints which prevent students working at height to access equipment.

\subsection{Supporting a Transition to Real Equipment}

Supporting a meaningful transition from a digital or simulated representation to the real context is difficult. Doing so flexibly [32] is the core challenge in ensuring students are able to make cognitive links between the representation and the real-world context. The equipment involved in stage lighting can be made available in certain configurations to students (e.g. one lamp at a time) to facilitate students in making this transition, but only if we can support the use of subsets of equipment that are manageable in the classroom.

\section{TOOLKIT DESIGN}

The design process consisted of three stages: i) the initial design and development of the toolkit by the authors (described in this section), ii) it's deployment in two UK high-schools as a working prototype (described in section 5 - Deployments) and iii) an evaluation with teachers and students who used the prototype (described in section 6 - Findings).

\subsection{The Toolkit}

In response to the challenges outlined in section 3, we designed a toolkit ${ }^{6}$ of resources, based around the use of tangible hardware proxies (see Figure 2). These proxies correspond to the literal physical properties (how light behaves), symbolic physical (they are individual 'units' that can be physically positioned relative to one another) whilst representing the key control characteristics of their

${ }^{6}$ https://github.com/digitalinteraction/gigacademy-microbit

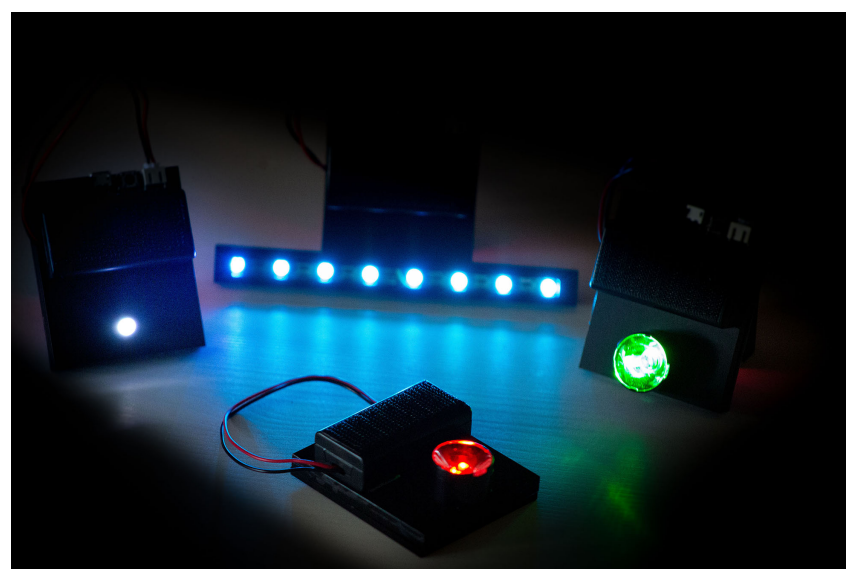

Figure 2: Left: Proxy 1, Middle Top: Proxy 3, Bottom + Right: Proxy 2 real counterparts (number of channels, channel mapping). We have used the term 'proxies' to refer to the hardware artifacts that were created as representations of stage lights within this toolkit. These differ from solutions offered in other models, simulations or virtual environments as they are designed specifically to:

- substitute one-to-one for real pieces of equipment, but without replicating the complexities of a high-fidelity simulation,

- model only the specific properties of the equipment that would enable skills based practice (e.g. optical properties, but not weight or physical size or cable complexity), whilst maintaining a direct association with the real equipment; and

- be incrementally switched out for real equipment, enabling larger scale simulations of venues with only a few pieces of 'real equipment' needing to be configured in the classroom, but without changing the operator experience of controlling the lamps.

Students use these proxies to represent real lamps of different types, interacting with them using either software or hardware based industry standard lighting controllers. By combining the proxies and real or software based control equipment students can practice different procedures and skills associated with stage lighting. A broker acts as a non-intrusive bridge between the proxy and real equipment to support fast and flexible configuration of practice environments. A model is used to represent the venue onto which lamps can be configured. The kit fits in a small plastic box which can be rapidly assembled by students in the classroom. A set of tasks scaffold student interaction with these elements.

\subsection{Proxy Lamps}

For our initial toolkit, we created proxies for three common lamp types available to schools. Each proxy lamp consists of a BBC Micro:bit ${ }^{7}$ running a Python application, placed inside a laser-cut plastic case. Each device is battery powered, and has one or multiple LEDs connected to the IO pins of the Micro:bit. On the reverse side of the Micro:bit (so that addresses can be adjusted whilst lamps are attached to the venue model), buttons adjust the starting address of the lamp and this is displayed on the screen. Each proxy lamp is controlled wirelessly. This is to avoid complex wiring, increase productive classroom time, and to reduce cognitive load, so that students can concentrate on the lamps and controller rather than specific cabling required.

4.2.1 Proxy 1: Single Channel Lamp. Historically, single channel lamps are passive (power to the lamp is controlled by a dimmmer which receives the DMX and is located elsewhere in the venue), containing a single bulb. The channel value controls the brightness of the bulb using a Pulse Width Modulator controlled by the received value. These lamps come in either 'profile' (focused spotlight) or 'wash' (unfocused floodlight). Light color can be changed by placing 'gels' (colored plastic) over the light source. The light beam can be adjusted using 'shutters' or 'barn-doors', shaping the pool of light the lamp creates on the stage. Our implementation consists of a single, white $5 \mathrm{~mm}$ LED with a $9^{\circ}$ angle of projection (see Figure 3 ). This produces a focused circle of white light which is congruent

\footnotetext{
${ }^{7}$ https://microbit.org/
} 
with the scale of the model. Cardboard or cellophane can be taped onto the unit to adjust color and experiment with shuttering.
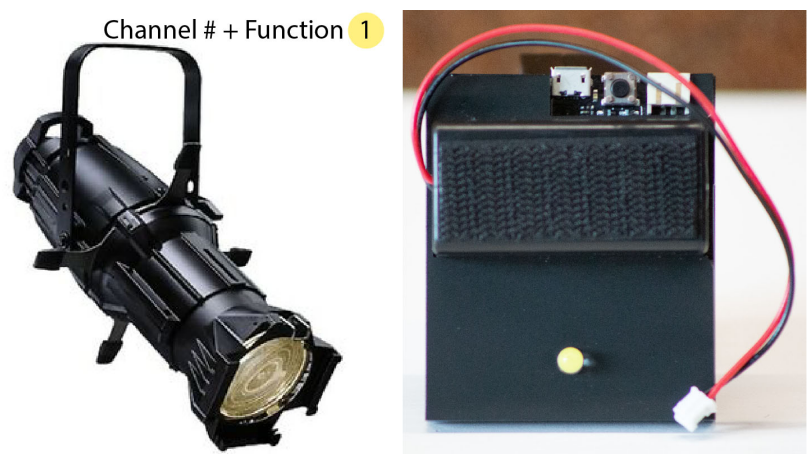

Figure 3: An ETC SourceFour (9kg) profile (left) and the corresponding proxy lamp (right)

4.2.2 Proxy 2: RGB Wash Lamp. The RGB wash lamp is an active lamp (DMX is received directly at the lamp) consisting of a single surface-mounted Neopixel RGB LED (see Figure 4). A lens is placed over the LED to replicate the optical characteristics of a full-size lamp, resulting in an un-focused circle of light at the venue models scale. Each color in the Neopixel is controlled by an individual channel, $1=$ Red, $2=$ Blue, $3=$ Green as a three channel personality. Setting these three levels to different values theoretically allows for 16387064 different colors to be produced by the lamp (see Figure 2).
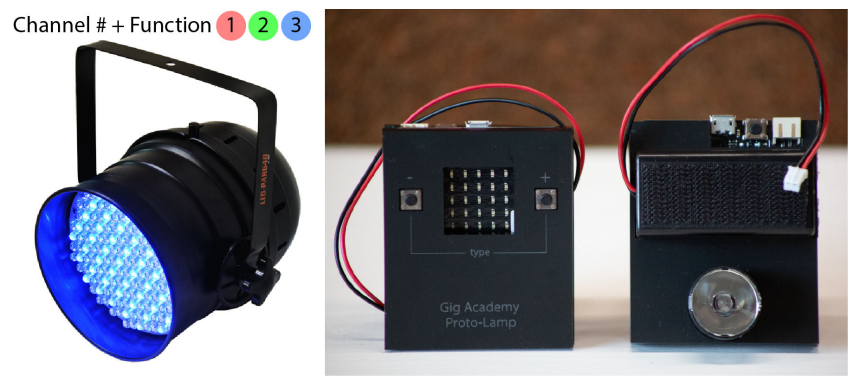

Figure 4: An AVE PAR64 (1kg) (left) and the corresponding proxy lamp (right)

4.2.3 Proxy 3: RGB Multi-Color Wash Lamp. Multiple RGB lamps are often combined in a single physical unit (see Figure 5). These are often used for special effects and animations as well as allowing for a larger stage coverage. A standard configuration consists of eight RGB lamps mounted linearly. Although it is one physical unit, each lamp it contains can be controlled as an individual RGB lamp in sequence (RGBRGB etc.). The specific brand of lamp we proxied also has a master brightness (1st channel) and effects control (last channel, not implemented) resulting in a 26 channel personality.

\subsection{Broker}

The requirement to seamlessly support students transition from abstract simplified models to real equipment led us to the development of the broker, the linchpin in the deployment of hardware
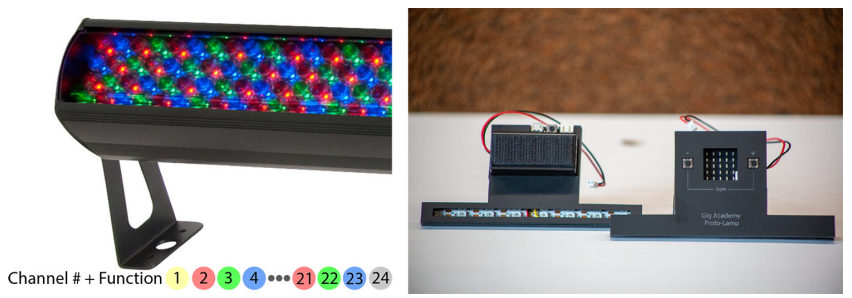

Figure 5: A Chauvet Color Rail (2.8kg) (left) and the corresponding proxy lamp (right)

proxies. The broker is a portable, mains-powered unit which acts as a bridge, or 'protocol broker', between the proxy lamps and existing industry stage lighting control protocols. This allows for both proxy lamps and real equipment to be controlled by both off-the-shelf software and industry standard hardware controllers. The broker allows students and educators to configure proxy lamps in different arrangements (Figure 6) depending on the learning context, classroom constraints and task objective. The broker allows the seamless integration of simulations (e.g. software virtual lighting environments), models (home-made venue models) and real hardware (stage lamps, control desks). Example configurations include:

(1) Using a software controller on a laptop to allow students to explore lamp properties, controlling proxy lamps on a classroom desk. (A->C)

(2) Learning to program the school's own hardware lighting desk ready for a show, by controlling proxy lamps in the classroom. (B->C)

(3) Using the software controller during a live show in the school venue using cues prepared in the classroom to control venue lighting. $(\mathrm{A}->\mathrm{D})$

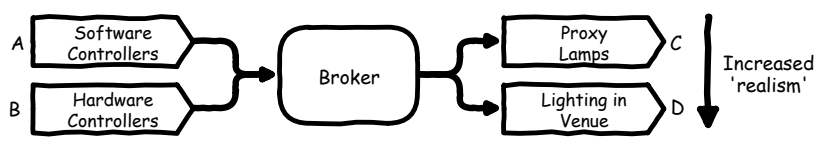

Figure 6: The broker facilitates multiple configurations of control and output

The broker contains a Raspberry Pi running Open Lighting Architecture $^{8}$ software. A Wi-Fi access point allows for connection to the broker from any control device in range using sACN, OSC or ARTNET protocols. To interface with existing physical equipment, two Enttec DMX USB Pro ${ }^{9}$ devices are attached to the Pi, for connecting DMX512 ${ }^{10}$ for DMX input and output. A Python script running on the PI receives universe updates from the OLA API and emits them via USB serial to a BBC Micro:bit connected via USB to the Pi. A Python script on the Micro:bit broadcasts this information via its native radio functionality to any Micro:bits in range, effectively generating a low-power wireless DMX universe. This universe can then be controlled by any software or hardware DMX

\footnotetext{
${ }^{8}$ https://www.openlighting.org/ola/

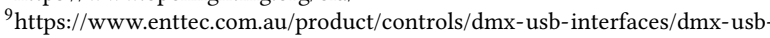
interface/

${ }^{10}$ https://en.wikipedia.org/wiki/DMX512
} 
lighting controller, or be mirrored by a physical DMX universe if real lamps are connected to the broker.

\subsection{Venue Model}

To support students experimentation with configuration of multiple lamps, including optical and visuospatial elements (such as ensuring suitable stage coverage and color mixing), we produced a simple wooden model (not to specific scale) representing the stage space in the school's venue (see Figure 8). The model included wooden dowels covered in Velcro in the relative position of the lighting bars on the venue's ceiling, allowing lamps to be easily re-positioned (with associated Velcro on the back of each lamp). Students are encouraged to use standard industry techniques such as mark making and taping on the model. The model is key in providing a shared common representation that supports groups of students working towards a common goal (the event they are planning) whilst engaging in different parts of their own learning journey.

\subsection{Task Scaffolding}

We designed seven tasks (Table 1) mapped to critical steps of practice that scaffold student engagement with different elements of both the technological and creative affordances of stage lighting, and the design skills required to utilize this technology effectively on the step. Each task is mapped to both the procedural knowledge required to perform a particular action in the stage lighting process and combinations of physical hardware and tangible proxies which best support developing the related skills. As task complexity increases, so does the potential complexity of equipment and processes required to engage in the learning. 'Real' equipment is incrementally added when specific focus is placed on practice with authentic hardware, in response to what might be available or what is practical within space and time constraints. Tasks were

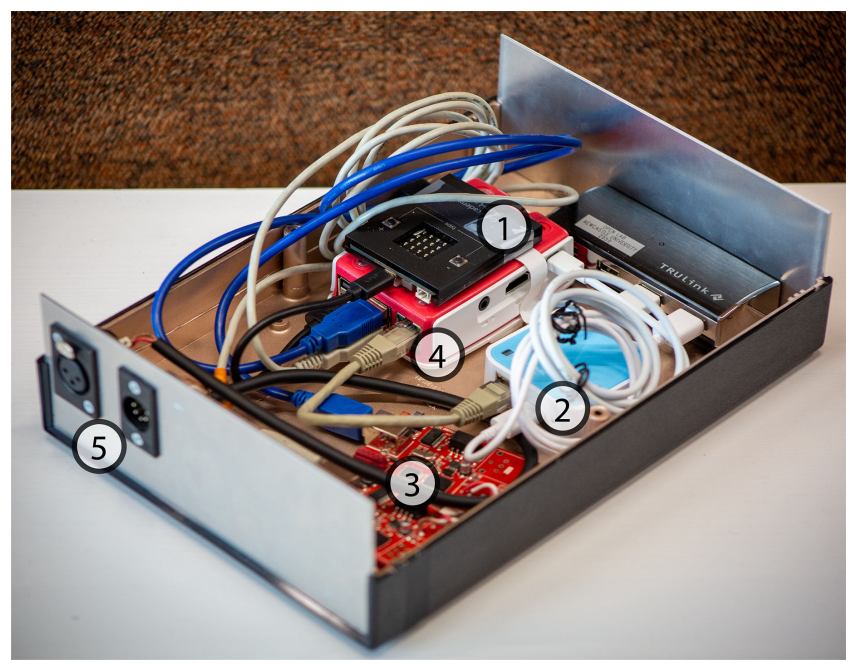

Figure 7: Prototype broker unit with lid removed. 1. Micro:bit, 2. WiFi router, 3. Enttec DMX Pro devices, 4. RaspberryPi, 5. DMX connectors designed to scaffold students working at their own pace, incrementally adding complexity whilst engaging them in operating both the models and the school's equipment. Tasks rely explicitly on the functionality of the broker in supporting a natural transition between proxy and authentic equipment. Thus, if a task places focus on the operation of the school's own lighting desk, there is no need to 'rig' an entire room with real stage lamps, but rather a number of proxies can be easily configured to visualize the current venue state and support practice using the desk. Conversely, if focus is being placed on the optical properties of real lamps, individual examples of these can be setup in the classroom, but be controlled by the much easier to setup - software controller.

\section{DEPLOYMENTS}

Our prototype was deployed in two public high-school (11-18yo) settings in the UK. In each school, a small group of students $(n=2$ in School One, and $n=4$ in School Two) were tasked with designing, planning, programming and operating the venue lighting rig for a live event at their school. In School One this was for a live music event, in School Two this was in preparation for a musical theater show. In each case the prototype toolkit was integrated into longitudinal (12 week) projects within the curriculum or extra-curricular groups. In both cases the teachers were working with a larger group of students to plan the whole live event with students split into smaller groups each with a particular focus such as lighting or sound. The students who chose to work on lighting were given the toolkit and worked mainly independently using the toolkit and resources to design, plan and program the lighting for the event.

Prior to working with us, neither school taught stage lighting as part of their curriculum. School One offered it as an extra-curricular

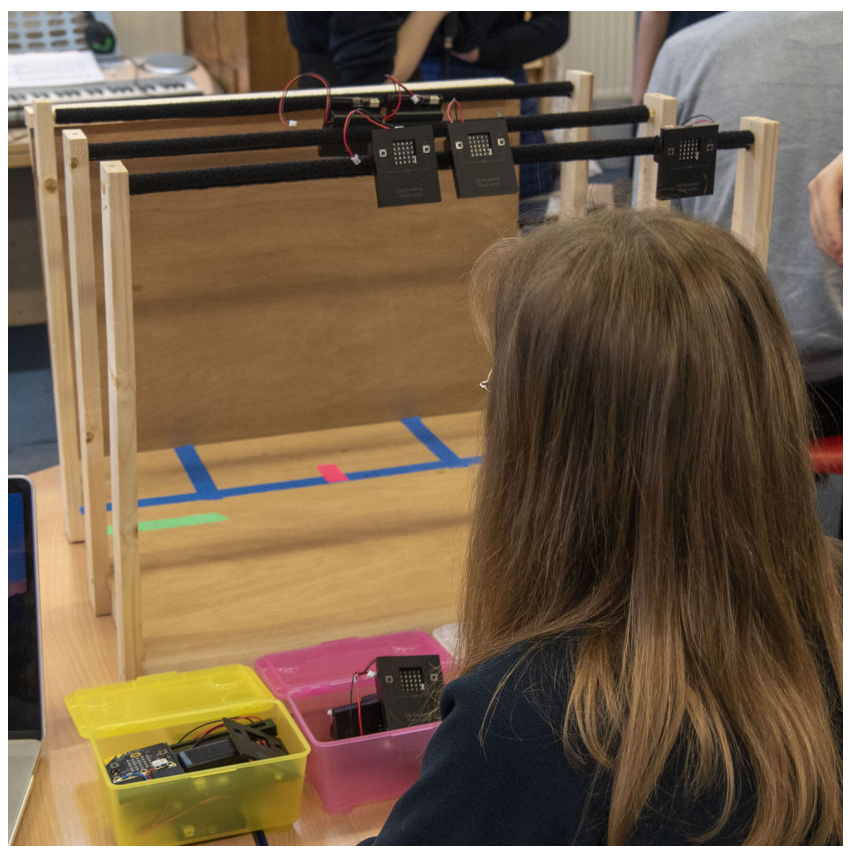

Figure 8: Students using venue model and proxy lamps to experiment with venue layouts 
Table 1: Table of design tasks and their mapping to the toolkit

\begin{tabular}{|c|c|c|c|c|}
\hline \# & Objective & Information & Task & Proxy Integration \& Transition \\
\hline 1. & $\begin{array}{l}\text { Understand DMX \& color mixing } \\
\text { Rig, Focus }\end{array}$ & $\begin{array}{l}\text { DMX protocol, channels, addresses, } \\
\text { color mixing, basic desk control }\end{array}$ & $\begin{array}{l}\text { Patch } 5 \text { lamps, address them cor- } \\
\text { rectly, create } 4 \text { different colors }\end{array}$ & $\begin{array}{l}1 \text { real lamp, } 5 \text { proxy lamps, hard- } \\
\text { ware controller }\end{array}$ \\
\hline 2. & $\begin{array}{l}\text { Understand how to shape light } \\
\text { Rig, Focus }\end{array}$ & $\begin{array}{l}\text { Stage coverage, terminology, color } \\
\text { washes, shapes, shuttering, barn } \\
\text { doors, gobos }\end{array}$ & $\begin{array}{l}\text { Patch in } 10 \text { lamps, address } 10 \text { lamps } \\
\text { correctly, position the lamps on the } \\
\text { rig, create a wash that covers the } \\
\text { whole stage }\end{array}$ & $\begin{array}{l}10 \text { proxy lamps, hardware con- } \\
\text { troller }\end{array}$ \\
\hline 3. & $\begin{array}{l}\text { Understand how light can be used } \\
\text { effectively } \\
\text { Plan, Rig, Focus }\end{array}$ & $\begin{array}{l}\text { Direction of lights, lighting people, } \\
\text { what can light do, mark out stage, } \\
\text { colors for moods }\end{array}$ & $\begin{array}{l}\text { design states for each item in the } \\
\text { script / running order }\end{array}$ & software controller, proxy lamps \\
\hline 4. & $\begin{array}{l}\text { Understand how to utilize lighting } \\
\text { control software } \\
\text { Configure, Program }\end{array}$ & Pre-sets, sequences, chases & $\begin{array}{l}\text { Program one of the scenes previ- } \\
\text { ously designed }\end{array}$ & software controller, proxy lamps \\
\hline 5. & $\begin{array}{l}\text { Understand industry standard plots } \\
\text { and plans } \\
\text { Plan, Configure }\end{array}$ & $\begin{array}{l}\text { Lighting plot, stage plan, cheat } \\
\text { sheet, plot sheet }\end{array}$ & $\begin{array}{l}\text { Produce the correct paperwork for } \\
\text { the previously designed scene you } \\
\text { designed earlier }\end{array}$ & hardware controller, proxy lamps \\
\hline 6. & $\begin{array}{l}\text { Understand re-usable states } \\
\text { Plan, Rig, Focus, Program }\end{array}$ & $\begin{array}{l}\text { Rig plan, program pre-sets, posi- } \\
\text { tioning, stage coverage, reusable } \\
\text { states }\end{array}$ & $\begin{array}{l}\text { Replicate the rig plan on the model, } \\
\text { create the scenes designed earlier } \\
\text { using lamps now defined on the rig } \\
\text { plan }\end{array}$ & hardware controller, proxy lamps \\
\hline 7. & $\begin{array}{l}\text { Understand pre-programming and } \\
\text { cues } \\
\text { Plan, Program }\end{array}$ & control of the desk, blackouts, cues & $\begin{array}{l}\text { Working from the final plans create } \\
\text { scenes and practice programming } \\
\text { the desk, practise moving between } \\
\text { scenes }\end{array}$ & $\begin{array}{l}\text { software controller, hardware con- } \\
\text { troller, proxy lamps }\end{array}$ \\
\hline
\end{tabular}

activity to one or two students who worked alongside the teacher in the lead up to school productions. This was typical of a masterapprentice model but was done entirely outside of curriculum times. We worked with that school as the teacher wanted to see how they could teach stage lighting as part of the performing arts curriculum within a classroom setting. School Two did not teach students stage lighting at all and was unsure how to do so either within the curriculum or as an extra-curricular activity given the constraints, particularly the health and safety concerns of working at height. We worked with that school as the teacher wanted to give more students the opportunity to be involved in the school production, beyond simply acting or singing on stage.

In both schools the toolkit was used during weekly (50min-2 hour) sessions either during class or in extra curricula time over a 12 week period leading up to the event. Each session was framed by the setting or continuation of one of the design tasks, and sessions were conducted without close supervision by teaching staff. Prior to each live event, the researchers, acting in the role of professional crew, helped the students rig, focus and address the lamps (in line with school health and safety policies) in accordance with the students' plan, and then supported them in programming and operating the lighting equipment during rehearsals and live show(s). We performed post-event interviews with the students ( 2 female, 4 male, median age 15) and the two teachers to explore their experiences of using the toolkit. This feedback, alongside our observations as educators in the context was analyzed through a reflexive thematic approach [4].

\section{FINDINGS}

\subsection{Proxies to Support Rapid Skill Development}

It was clear from the feedback that the proxies allowed students to build familiarity with the the control equipment (both hardware and software) that was available to them in the school:

The model lights were good because they just let us look at the different settings and helped us also understand the software that we were using. (S2)

Students also commented that the proxies were key in them building up a clear cognitive model of the properties of each lamp, and of the potential configuration of the venue.

It helped us to understand what each type of light would do and it just helped understand what set up we would use. (S2)

More importantly for supporting independent learning through practical experience, students reported that the proxies enabled them to foster their creativity and experiment with possible configurations.

Using the model lights that's really when we came up with ideas about how we should set it [the lighting rig] out. (S1)

Indeed, the proxies formed an integral part of the learning process for students utilizing the proxy and broker in some way during every session. After using the model of the venue during earlier steps, members of the team would refer back to configurations as a shared group representation, facilitating communication and discussion when creating plans for the rig (see Figure 8). 
When asked to suggest changes to the process during postevaluation, students expressed the desire for models that acted as proxies for more of the equipment they had access to (in the prototype, no proxies were made to represent the more advanced moving-head lamps, or effects devices such as smoke machines). It was clear that they valued the physical representation of the hardware which allowed them to reconcile the digital representation within the lighting control system, leading them to understand both.

...so we could understand the moving heads a bit more because on the day [of the event] we struggled ... so if we had models of them we could just understand the software for moving heads better. (S1)

Students demonstrated that they were able to transfer their skills acquired using the proxies to the full sized lighting rig on event day in both contexts. When speaking to one of the teachers from the first school, following the technical and dress rehearsals, they commented that the lighting team's confidence was impressive and admitted to having been skeptical that students would be able to run the show. They expected that the authors (who were there to support the students) would really be in control and operating all equipment while the students would observe. (taken from observation notes). Students did indeed respond to the challenges presented to them on event day, in both cases helping to rig, focus and cable the lighting rig on the day of the event to the plan they had prepared (with help from the authors for Health and Safety reasons). During each event, the first author (an experienced lighting technician) did not have to intervene or override any student decisions or assist with any operational issues.

\subsection{Practicality in the Classroom}

A key design requirement of our resource was to enable skills learning in a classroom setting. On the form factor of the proxies, one teacher commented,

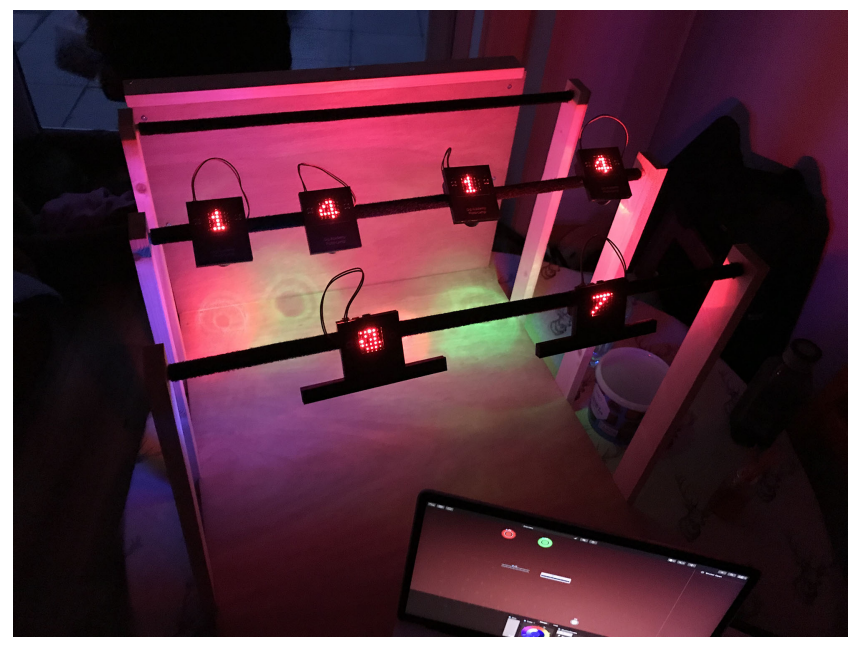

Figure 9: Students using venue model and proxy lamps to program cues into their school lighting desk ready for the show
They were a good size for students to work with, they were big enough that they weren't too fiddly but small enough to be able to move around easily without worrying about weight or practicalities. (T2)

Due to their portability, students were able to move them between classrooms week-to-week. At various points they were observed actively changing spaces during classes either in response to space constraints or to demonstrate their skills to other students. Additionally, teachers were impressed that no specialist rooms needed booking (i.e. workshop, computer room, event space) to make it possible for the students to learn these skills.

It is difficult for us [to book the hall] as the hall is used for break/lunch (especially with a split lunch it was often during their lessons). This [using the lights] was considerably better than having to go and use the big ones every lesson...and manageable as well. (T2)

The teacher felt that using the proxies made teaching stage lighting practical (and more manageable) as using the the models allowed her to keep students within the classroom rather than splitting her supervision between multiple spaces within the school (as would be the case if they were limited to a specific event space), commenting that

[The lights were] much easier as I knew where [the students] were and had less running around the school to do to check on everyone, it then comes down to supervision - having kids spread out around the school is always a bit impractical. (T2)

This again highlights the importance teachers place on the logistics of teaching spaces and resources.

\subsection{Supporting Scaffolded Practice}

When discussing the benefits of the proxies for students one teacher commented,

They probably did get more practice time and it also added a 'stepped' approach to using equipment that they found quite scary. (T2)

This evidence of the proxies scaffolding a 'stepped' approach aligns with our goal of supporting students incrementally learning skills by allowing the students to gradually increase the possible complexity of the equipment at their own pace (transitioning). Students were observed spending a minimal amount of time getting the models out and putting them away in each lesson. This selfserve approach allowed students to use the equipment in every lesson, and also increase the amount of time and opportunities to practice their design, configuration, and programming skills, as one teacher comments,

This stepped approach gives them more opportunities to develop the skills rather than throwing them straight in the deep end which might just scare them off rather than get them excited. (T2)

A particular example of this was seen when students were learning to use moving-head lamps (lights that both pan and tilt as well as have various color and shape options). At present our prototype does not proxy such a lamp, so at various points they were given access to a single full-scale lamp in the classroom. In these cases, 
students required 20 minutes to un-box, hang the lamp on a lighting stand and connect to the broker before even being able to operate it utilizing the software. In light of this, students commented that 'the models are so much easier, just take them out of the box, plug the battery in and go (Observation Notes)'.

\subsection{Building Confidence}

Confidence is key for both students and teachers in the learning environment, particularly when engaging with skills and knowledge that are new to the teacher. The teachers we worked with felt that the proxies gave them the confidence to teach stage lighting to students on their own, with one teacher commenting that it was

less terrifying for everyone, [it would] all come down to my confidence - I think the kids really enjoyed it and got a lot out of it so if I felt confident and knew that we had suitable kit I would definitely do it. (T2)

More specifically, teachers raised concerns about the perceived risk in exposing students to valuable school assets (i.e. equipment and venue), commenting that

The smaller scale (less expense, less chance of damage, no big lights zooming across the classroom drawing attention to them, etc) is definitely appealing when students are finding their feet. (T2)

In terms of building student confidence, one reason they felt the proxies were advantageous was in making students feel more confident in experimentation with the equipment, especially in the early stages of learning

If something was to go wrong you don't have this big light flying around the classroom or anything that everyone can see and everyone is looking at and you're like 'oh my god, it's going wrong' it was quite quirky to have these cute little lights, I think it probably would have worked a lot better than not having had them. (T2)

A highlighted concern here being the perception by students as 'getting it wrong' in a room of their peers. As the proxies were not the 'real' equipment, there was less concern over the visibility of perceived mistakes. In addition to the perceived risk of mistakes, teachers were also relieved by the low chance (and resulting cost) of breakages using the proxies compared to the full-size equipment.

\section{DISCUSSION}

Our experience in designing a set of resources and the reactions of both students and teachers revealed key insights about the value of using tangible proxies to support the teaching of practice-based skills.

\subsection{Supporting The Teaching of Practice-Based Skills through Risk Reduction}

Our findings indicate that the use of tangible proxies to scaffold the learning process reduces both the real and perceived risk for teaching staff. We saw that this lead to teachers feeling that they were more likely to engage with stage lighting as an option within curricula.
The size of the proxies mean that students can use them within a more traditional and controlled environment - the classroom, rather than the venue. This move back into a traditional learning space puts the teacher back in a position of control, allowing them to focus on student learning rather than logistical and health and safety concerns. Secondly, schools are also keen to protect their capital investments, leading to an assumption by teachers that students' interaction with expensive equipment should be limited and done under close supervision. Our experience suggests that this assumption is unfounded, however it was a concern of the teachers we worked with. Use of the proxies gave students the opportunity to 'prove' their skills and ownership of the creative process which ultimately mitigated the perceived risk of miss-use of expensive equipment. In this way trust was built between teacher and student which meant that teachers were more willing to allow students to utilize the full size equipment autonomously for the final event. Indeed, students demonstrated competency and skill when presented with the opportunity to operate and configure the real equipment, relishing the trust given to them by the teachers. More fundamentally, by brokering trust between teacher and students, proxies support teaching pedagogies better suited to practice-based skills.

\subsection{Supporting Flexible Reconfiguration for Conceptual Learning}

Although we designed the broker to allow for rapid and flexible reconfiguration of controllers and outputs through experimentation, we were open to how these were appropriated within the classroom setting. The high-level design tasks which necessitated interaction with the proxies did structure deliberate practice [9] with specific tasks aimed at specific gaps in knowledge and specific deliverables, which facilitated immediate feedback. These tasks were designed to allow students to progress at their own pace (most often without any teacher initiation) and transition between simulation and real equipment where using specific hardware was appropriate. The

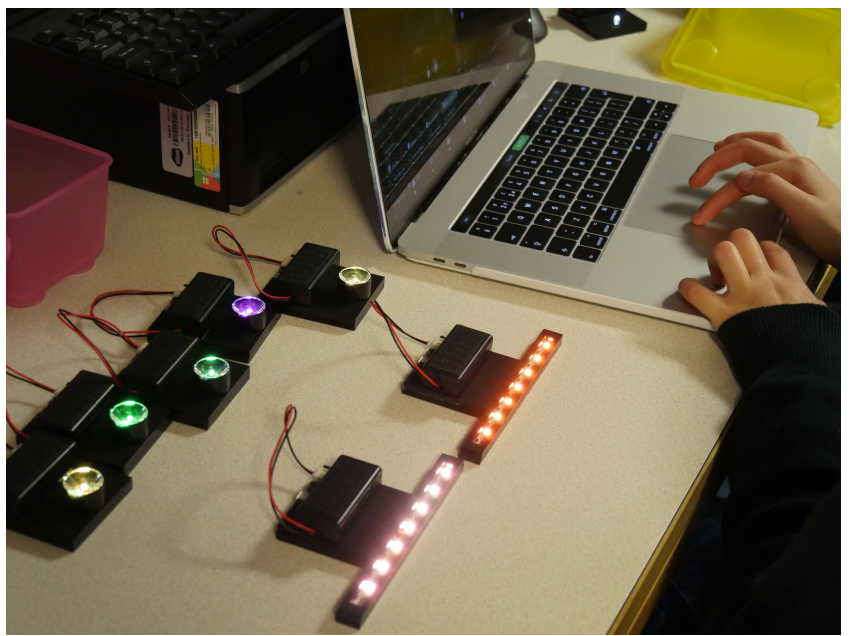

Figure 10: A student explores the different properties of the lamps and how they map to the software lighting controller 
broker acted as the anchor which allowed for a graduated transition between software controllers (better for learning lighting design and rig configuration), hardware controllers (which more commonly exist in the school's venues), prototype lamps (which are easy to move around and configure), and real lamps (which are more difficult to manipulate). As students increased in their expertise, they graduated in the complexity of the actions they were performing, from interactions with single lamps to understand their properties, through to programming complex scenes using industry standard equipment. The use of proxies with structured tasks supported independent learning [48] without direct access to experts, thus allowing for the development of these skills in the classroom.

The use of such tangible proxies also allowed students to rapidly engage in experimentation, which through practical embodied engagement allowed them to develop the requisite mental models of how the equipment operates in multiple configurations and settings. It is only through supporting such rapid iteration that students were provided with opportunities for developing these models. In contrast to traditional master-apprentice learning, the form factor and external visibility of the tangible proxies facilitated peer and group learning that would not otherwise be possible. The proxies allowed students to experiment with equipment, (particularly moving lamps around), in ways that would be impossible in a real venue due to health and safety risks. This allowed them to build up a clear understanding of both the technical limitations and creative possibilities. This ability to experiment allowed students to form confidence in handling errors. For example, as the proxies behaved in the same way as the physical lamp would in the rig, any errors such as addressing the channel incorrectly were immediately visible and students were able to experiment with solutions quickly and easily by directly adjusting the configuration of the proxies to better understand the underlying concepts. In a real-world environment changing lighting channels would have involved working at height to adjust lamp settings which is both time consuming and dangerous.

The use of the broker to enable model and real lights to be switched out quickly and easily meant that the mapping from representation to real-world object could be tested and understood quickly and easily. This reduced the cognitive load required to process the change from representation to real-world scenario and ensured that students were able to transfer skills learned from using the models to these real-world scenarios with stage lighting.

\subsection{The Broker as a Route to New Pedagogies}

Designing hardware proxies to support practice in these scenarios involves: i) identifying specific skills that need to be supported in deliberate practice, ii) mapping the properties of 'real' equipment that need to be represented in models and the constraints of using the real equipment in the classroom to support a fluid learning transition and iii) identifying which control or representation protocols the broker needs to support in order to support the practice of these skills. In our implementation we focused on reducing the physical size of stage lighting hardware, reducing setup time and mitigating risk for students whilst mapping the optical, configuration and visuospatial characteristics of stage lamps. In this context the broker enabled direct connection and mirroring of lighting control between industry standards and models.

Without the broker, tangible proxies are just low-fidelity models of stage lighting equipment. Although, in isolation, they support some elements of practice-based learning (such as visuospatial configuration), it is the broker that ultimately enables students' deliberate practice and independent graduated transition from models to real equipment. The broker provides a bridge between industry standard software inputs and hardware proxies as outputs, allowing students opportunities for deliberate practice while mitigating both the risk and expense of using large and potentially dangerous equipment. Outside of the creative arts, vocational roles which include skills based on systems or control such as electrical wiring, building management, plumbing, assembly lines etc. are obvious contexts in which key skills based practices could be mapped to classroom based activities through the use of hardware proxies.

The ability to offer this bridge means that the use of a broker then supports the design of authentic practice-based curricula by allowing the flexible reconfiguration of a mix of real and model equipment in the classroom. This dynamic transition between model and real equipment is only possible due to the design of the broker, that specifically supports both real and model representations working together. The combination of tangible proxies together with a broker can be applied to other domains such as learning to operate $\mathrm{CNC}$ machinery or industrial scale robotics - where working with industry standard equipment can be prohibitively expensive and/or dangerous.

Interestingly, we discovered that the broker opens up new opportunities for developing innovative pedagogies within the classroom. In addition to providing creative opportunities through the reduction of risk, the broker enabled multiple students to interact simultaneously (something not possible using traditional lighting control equipment). The broker allows multiple students to interact with a single representation of the venue, but using a mix of different controllers, models and real equipment simultaneously. It is well understood that co-located collaborative environments which present a shared representation of a problem space foster peer-learning [19], through negotiation and public expression of intent [15]. In practice-based settings this type of learning is not possible due to the single-user limitations of the equipment, but can be encouraged by using tangible proxies.

\section{CONCLUSION}

In this paper we presented the design of a tangible toolkit to support the teaching of practice-based skills in the K-12 classroom. We developed a toolkit consisting of tangible proxies that embody key elements of lighting equipment and a broker, which supports the transition from tangible models and simulations to real equipment, within the constraints of a classroom environment. We contribute considerations for designing such proxies through the notion of a 'broker'. This broker allows for flexible reconfiguration of equipment to support students conceptual learning as well as opening up opportunities for collaborative pedagogies in the teaching of technical stage roles within the Creative Arts in schools. Our findings suggest that such proxies offer the potential to engage students in 
practice-based skills learning which was previously impractical in institutional contexts.

\section{ACKNOWLEDGMENTS}

This research was funded by the EPSRC Digital Economy Research Center (EP/M023001/1) and the EPSRC CDT in Digital Civics (EP/L016176/1).

\section{REFERENCES}

[1] Saskia Bakker, Elise van den Hoven, and Alissa N Antle. 2010. MoSo Tangibles: Evaluating Embodied Learning. In TEI 2010 (TEI '11). Association for Computing Machinery, New York, NY, USA, 85-92. https://doi.org/10.1145/1935701.1935720

[2] Tom Bartindale and Patrick Olivier. 2013. ThorDMX. In CHI 2013 Extended Abstracts. ACM Press, New York, New York, USA, 3019. https://doi.org/10.1145/ 2468356.2479600

[3] Dirk Börner, Bernardo Tabuenca, Jeroen Storm, Sven Happe, and Marcus Specht. 2015. Tangible Interactive Ambient Display Prototypes to Support Learning Scenarios. In TEI 2015. Association for Computing Machinery, New York, NY, USA, 721-726. https://doi.org/10.1145/2677199.2687908

[4] Virginia Braun and Victoria Clarke. 2006. Using thematic analysis in psychology. Qualitative Research in psychology2 3, 2 (2006), 77-101.

[5] Drew Campbell. 1999. Technical theater for nontechnical people. Choice Reviews Online 37, 04 (1999), 37-2092. https://doi.org/10.5860/choice.37-2092

[6] Emily S. Cramer and Alissa N. Antle. 2015. Button Matrix: How tangible interfaces can structure physical experiences for learning. In TEI 2015. Association for Computing Machinery, Inc, New York, New York, USA, 301-304. https://doi. org/10.1145/2677199.2680566

[7] Sébastien Cuendet, Engin Bumbacher, and Pierre Dillenbourg. 2012. Tangible vs Virtual representations: When tangibles benefit the training of spatial skills. In NordiCHI 2012. ACM Press, New York, New York, USA, 99-108. https://doi.org/ $10.1145 / 2399016.2399032$

[8] Clifford De Raffaele, Serengul Smith, and Orhan Gemikonakli. 2016. The aptness of Tangible User Interfaces for explaining abstract computer network principles. In Proceedings - Frontiers in Education Conference, FIE, Vol. 2016-November. Institute of Electrical and Electronics Engineers Inc. https://doi.org/10.1109/FIE 2016.7757573

[9] K Anders Ericsson, Ralf Th Krampe, and Clemens Tesch-Römer. 1993. The role of deliberate practice in the acquisition of expert performance. Psychological Review 100, 3 (1993), 363-406. https://doi.org/10.1037/0033-295x.100.3.363

[10] Alison Fuller and Lorna Unwin. 2009. Change and continuity in apprenticeship: the resilience of a model of learning. Fournal of Education and Work 22, 5 (11 2009), 405-416. https://doi.org/10.1080/13639080903454043

[11] Sidhant Goyal, Rohan S Vijay, Charu Monga, and Pratul Kalita. 2016. Code Bits An Inexpensive Tangible Computational Thinking Toolkit For K-12 Curriculum. In TEI 2016 (TEI '16). Association for Computing Machinery, New York, NY, USA, 441-447. https://doi.org/10.1145/2839462.2856541

[12] Teodor P Grantcharov and Richard K Reznick. 2008. Teaching procedural skills. , 1129-1131 pages. https://doi.org/10.1136/bmj.39517.686956.47

[13] G James Greeno. 1989. Situations, mental models, and generative knowledge. In Complex information processing: The impact of Herbert A. Simon. 285-318.

[14] Shuchi Grover and Satabdi Basu. 2017. Measuring student learning in introductory block-based programming: Examining misconceptions of loops, variables, and Boolean logic. In ITiCSE (SIGCSE '17). Association for Computing Machinery, New York, NY, USA, 267-272. https://doi.org/10.1145/3017680.3017723

[15] Carl Gutwin and Saul Greenberg. 1998. Design for individuals, design for groups. In CSCW 1998. ACM Press, New York, New York, USA, 207-216. https://doi.org/ 10.1145/289444.289495

[16] Bart Hengeveld, Caroline Hummels, Kees Overbeeke, Riny Voort, Hans van Balkom, and Jan de Moor. 2009. Tangibles for Toddlers Learning Language. In TEI 2009 (TEI '09). Association for Computing Machinery, New York, NY, USA, 161-168. https://doi.org/10.1145/1517664.1517702

[17] Eva Hornecker. 2011. The role of physicality in Tangible and embodied interactions. Interactions 18, 2 (3 2011), 19-23. https://doi.org/10.1145/1925820.1925826

[18] Tom Jenkins and Ian Bogost. 2015. Escaping the sandbox: Making and its future. In TEI 2015. 29-32. https://doi.org/10.1145/2677199.2680558

[19] Ahmed Kharrufa, David Leat, and Patrick Olivier. 2010. Digital mysteries. In ITS 2010. ACM Press, New York, New York, USA, 197. https://doi.org/10.1145/ 1936652.1936689

[20] Robert Klingelhoefer. 2016. The craft and art of scenic design: Strategies, concepts, and resources. 1-285 pages. https://doi.org/10.4324/9781315676173

[21] Roger Kneebone. 2005. Evaluating clinical simulations for learning procedura skills: A theory-based approach. , 549-553 pages. https://doi.org/10.1097/ 00001888-200506000-00006
[22] David Kolb. 2000. The Process of Experiential Learning. In Strategic Learning in a Knowledge Economy. Elsevier, 313-331. https://doi.org/10.1016/b978-0-75067223-8.50017-4

[23] Stacey Kuznetsov, Piyum Fernando, Emily Ritter, Cassandra Barrett, Jennifer Weiler, and Marissa Rohr. 2018. Screenprinting and TEI: Supporting engagement with STEAM through DIY fabrication of smart materials. In TEI 2018, Vol. 2018Janua. 211-220. https://doi.org/10.1145/3173225.3173253

[24] Hideaki Kuzuoka, Naomi Yamashita, Hiroshi Kato, Hideyuki Suzuki, and Yoshihiko Kubota. 2014. Tangible earth: Tangible learning environment for astronomy education. In HAI 2014. Association for Computing Machinery, Inc, New York, New York, USA, 23-27. https://doi.org/10.1145/2658861.2658870

[25] John Maeda. 2013. Stem+ art= steam. The STEAM journal 1, 1 (2013), 34

[26] Andrew Manches, Claire O'Malley, and Steve Benford. 2009. Physical Manipulation: Evaluating the Potential for Tangible Designs. In TEI 2009 (TEI '09). Association for Computing Machinery, New York, NY, USA, 77-84. https: //doi.org/10.1145/1517664.1517688

[27] Paul Marshall. 2007. Do tangible interfaces enhance learning?. In TEI 2007. ACM Press, New York, New York, USA, 163-170. https://doi.org/10.1145/1226969. 1227004

[28] Yoshiaki Matsuzawa, Takashi Ohata, Manabu Sugiura, and Sanshiro Sakai. 2015. Language migration in non-CS introductory programming through mutual language translation environment. In SIGCSE 2015. 185-190. https://doi.org/10. $1145 / 2676723.2677230$

[29] Edward F. Melcer and Katherine Isbister. 2016. Bridging the physical divide: A design framework for embodied learning games and simulations. In CHI 2016, Vol. 07-12-May-2016. Association for Computing Machinery, New York, New York, USA, 2225-2233. https://doi.org/10.1145/2851581.2892455

[30] Diana Nowacka and David Kirk. 2014. Tangible autonomous interfaces (TAIs): Exploring autonomous behaviours in TUIs. In TEI 2014. Association for Computing Machinery, New York, New York, USA, 1-8. https://doi.org/10.1145/2540930. 2540942

[31] Sara Price. 2008. A representation approach to conceptualizing tangible learning environments. In TEI 2008. ACM Press, New York, New York, USA, 151-158. https://doi.org/10.1145/1347390.1347425

[32] Sara Price and Taciana Pontual Falcão. 2009. Designing for physical-digital correspondence in tangible learning environments. In IDC 2009. ACM Press, New York, New York, USA, 194-197. https://doi.org/10.1145/1551788.1551826

[33] Sara Price, Yvonne Rogers, Stanton Danae Scaife, Mike, and Helen Neale. 2003. Using 'tangibles' to promote novel forms of playful learning. Interacting with Computers 15, 2 (4 2003), 169-185. https://doi.org/10.1016/S0953-5438(03)00006-7

[34] Francis Reid. 1998. Discovering stage lighting. (1998). https: //content.taylorfrancis.com/books/download?dac $=$ C2012-0-053030\&isbn $=9781136084225 \&$ format $=$ googlePreviewPdf

[35] Yvonne Rogers, Mike Scaife, Silvia Gabrielli, Hilary Smith, and Eric Harris. 2002. Presence: Teleoperators and Virtual Environments. Vol. 11. Chapter A conceptual framework for mixed reality environments: Designing novel learning activities for young children, 677-686. https://doi.org/10.1162/105474602321050776

[36] Bertrand Schneider, Patrick Jermann, Guillaume Zufferey, and Pierre Dillenbourg. 2010. Benefits of a tangible interface for collaborative learning and interaction. IEEE Transactions on Learning Technologies 4, 3 (2010), 222-232.

[37] Donald A Schon. 1984. The Reflective Practitioner: How Professionals Think In Action. Basic Books. https://books.google.com.au/books?id=ceJIWay4-jgC

[38] Lawrence Shapiro. 2019. Embodied cognition. Routledge.

[39] Steven Louis Shelley. 2013. A Practical Guide to Stage Lighting. Routledge. https://doi.org/10.4324/9780080879062

[40] Adalberto L Simeone, Eduardo Velloso, and Hans Gellersen. 2015. Substitutional reality: Using the physical environment todesign virtual reality experiences. In CHI 2015, Vol. 2015-April. 3307-3316. https://doi.org/10.1145/2702123.2702389

[41] Larisa Sitorus, Shan Shan Cao, and Jacob Buur. 2007. Tangible User Interfaces for Configuration Practices. In TEI 2007 (TEI '07). Association for Computing Machinery, New York, NY, USA, 223-230. https://doi.org/10.1145/1226969.1227015

[42] David Throsby and Katya Petetskaya. 2017. Making Art Work: An Economic Study of Professional Artists in Australia. Australia Council for the Arts. 244 pages. https://researchers.mq.edu.au/en/publications/making-art-work-aneconomic-study-of-professional-artists-in-aust

[43] Brigg Ullmer and Hiroshi Ishii. 2000. Emerging frameworks for tangible user interfaces. IBM Systems fournal 39, 3-4 (2000), 915-930. https://doi.org/10.1147/ sj.393.0915

[44] John Underkoffler and Hiroshi Ishii. 1998. Illuminating light. In CHI 1998. Association for Computing Machinery (ACM), New York, New York, USA, 542-549. https://doi.org/10.1145/274644.274717

[45] United Nations. 2018. Trends in international trade in creative industries 2012-2015. Technical Report. 445 pages. https://unctad.org/en/pages/PublicationWebflyer. aspx?publicationid $=2328$

[46] Christina Vasiliou, Andri Ioannou, and Panayiotis Zaphiris. 2014. Understanding collaborative learning activities in an information ecology: A distributed cognition account. Computers in Human Behavior 41 (12 2014), 544-553. https://doi.org/10.1016/j.chb.2014.09.057 
[47] Etienne Wenger. 1998. Communities of Practice. Learning, Meaning and Identity. Cambridge University Press.

[48] David Wood, Jerome S. Bruner, and Gail Ross. 1976. The Role of Tutoring in Problem Solving. Fournal of Child Psychology and Psychiatry 17, 2 (1976), 89-100. https://doi.org/10.1111/j.1469-7610.1976.tb00381.x
[49] Anna Xambó, Brigid Drozda, Anna Weisling, Brian Magerko, Marc Huet, Travis Gasque, and Jason Freeman. 2017. Experience and ownership with a tangible computational music installation for informal learning. In TEI 2017 (TEI '17). Association for Computing Machinery, New York, NY, USA, 351-360. https: //doi.org/10.1145/3024969.3024988 